1999-06-01

\title{
Model Predictive Satisficing Fuzzy Logic Control
}

Richard L. Frost

Michael A. Goodrich

mike@cs.byu.edu

Wynn C. Stirling

wynn_stirling@byu.edu

Follow this and additional works at: https://scholarsarchive.byu.edu/facpub

Part of the Computer Sciences Commons

\section{Original Publication Citation}

M. A. Goodrich, W. C. Stirling, and R. L. Frost. Model Predictive Satisficing Fuzzy Logic Control. IEEE Transactions on Fuzzy Systems v.7, no.3:319-322, June 1999.

\section{BYU ScholarsArchive Citation}

Frost, Richard L.; Goodrich, Michael A.; and Stirling, Wynn C., "Model Predictive Satisficing Fuzzy Logic Control" (1999). Faculty Publications. 614.

https://scholarsarchive.byu.edu/facpub/614

This Peer-Reviewed Article is brought to you for free and open access by BYU ScholarsArchive. It has been accepted for inclusion in Faculty Publications by an authorized administrator of BYU ScholarsArchive. For more information, please contact ellen_amatangelo@byu.edu. 


\title{
Model Predictive Satisficing Fuzzy Logic Control
}

\author{
Michael A. Goodrich, Wynn C. Stirling, and Richard L. Frost
}

\begin{abstract}
Model-predictive control, which is an alternative to conventional optimal control, provides controller solutions to many constrained and nonlinear control problems. However, even when a good model is available, it may be necessary for an expert to specify the relationship between local model predictions and global system performance. We present a satisficing fuzzy logic controller that is based on a receding control horizon, but which employs a fuzzy description of system consequences via model predictions. This controller considers the gains and losses associated with each control action, is compatible with robust design objectives, and permits flexible defuzzifier design. We demonstrate the controller's application to representative problems from the control of uncertain nonlinear systems.
\end{abstract}

Index Terms - Decision-making, intelligent control, predictive control, satisficing.

\section{INTRODUCTION}

A LTHOUGH many useful optimality-based controller designs exist, it is sometimes difficult to define and find optimal solutions to highly nonlinear highly complex problems. This places controller design for such systems in the class of ill-formed problems wherein there is a lack of sufficient information, time, or resources to define or to find the optimal solution [1]. Ill-formed problems motivate the search for intelligent solutions, the success of which rests, to some degree, upon the belief that finding the optimal decision is not necessary for making justifiable decisions [2]-[4]. The search for intelligent solutions necessarily addresses: 1) the definition and computation of acceptable solutions; 2) the identification of models (whether implicit in expert rules or explicit in differential equations); and 3) the robust synthesis of information from multiple sources. To generate intelligent controllers, each issue in this noninclusive list demands a formal and justifiable treatment. In this paper, we address these issues from a perspective that employs strongly satisficing decision theory and fuzzy logic.

\section{A. Solution Motivation and Background}

In conventional optimal control, explicit models describe possible system consequences and these possible consequences are ordered using a cost function. For well-formed problems, minimizing this cost function determines the optimal global solution with respect to the specified cost function and the

Manuscript received March 27, 1998; revised July 31, 1998. This work was supported in part by Nissan Cambridge Basic Research, Nissan Research and Development, Inc.

M. A. Goodrich is with Nissan Cambridge Basic Research, Cambridge, MA 02142 USA.

W. C. Stirling and R. L. Frost are with the Electrical and Computer Engineering Department, Brigham Young University, Provo, UT 84602 USA.

Publisher Item Identifier S 1063-6706(99)04934-6.

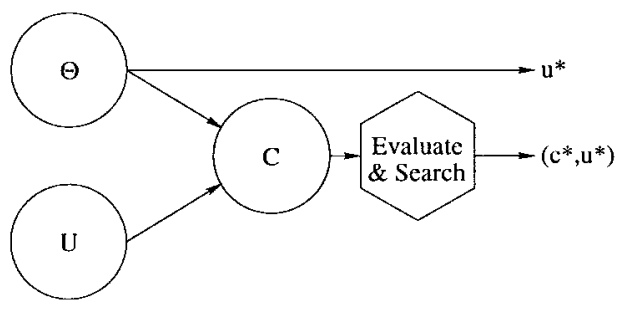

Fig. 1. Steps of inference: observation to action.

given explicit model. Specifying the cost function is left to the designer (an implicit expert); from experience, quadratic costs are often used because they yield computable solutions and, in the case of positive definite cost matrices, produce unique minimizing solutions.

In conventional fuzzy logic control, the predicted system consequences are implicit in the rules, where the model is implicit in the rules too. Rules are obtained explicitly from an expert and presumably have been compiled from the following sequence of inferences (see Fig. 1):

1) if control $u \in U$ is applied given observation $\theta \in \Theta$, then the plant consequence is $c(\theta, u) \in C$;

2) given observation $\theta$, plant consequence $c^{*}=$ $\max _{u} c(\theta, u)$ is most desirable (i.e., maximizing);

3) given observation $\theta$, control $u^{*}=\arg _{u \in U}\left\{c(\theta, u)=c^{*}\right\}$ should be used;

4) given observation $\theta$, do $u^{*}$.

Since expert rules are not easily obtained for all $\theta$, fuzzy logic methods are used to interpolate among a grid of selected $\theta$ points.

Conventional optimal control assumes not only that an explicit model of the plant exists, but also that an implicit expert is available to prescribe a cost function that can be solved using optimization methods. Though frequently effective for controller design, some problems are not appropriately addressed by optimality-based methods [5]. In contrast to optimal control, conventional fuzzy logic control assumes that an explicit expert exists who can construct a rule base that transforms observations into plant controls via an implicit model of the system. Again, though frequently effective for controller design, useful information may be unnecessarily discarded by compiling steps 1)-3) into 4).

There exist problems that can benefit from the best of both optimal and fuzzy approaches to controller design. For example, the use of local model predictions may free the expert from some of the burden of performing mental simulations of the plant [6] and instead allow the expert to identify consequences as beneficial or costly. This paper is interested in a subset of such problems where an explicit model of 
the system exists and an explicit expert is used to transform local model predictions into global evaluations of gains and losses. Such an approach is necessary when complexity and uncertainty prevent precise predictions about global plant behavior, but when useful information is available from local plant predictions.

Evaluating the gains and losses of a control action using model predictions is based on the comparative "cost/benefit" structure of strongly satisficing decision theory (SSDT) [4], [7], [8]. SSDT modifies the objective of finding the optimal control (with respect to a given model structure and cost function) by including the less ambitious (and perhaps more robust) objective of avoiding error [4]. This design paradigm employs the comparative rationality that is suggested by Simon's satisficing principle [9], [10], the domination principle from multi-attribute utility theory and the mathematics of Levi's error avoidance principle [11]. Employing fuzzy logic in the synthesis of SSDT-based model predictive controllers produces a method for systematically designing fuzzy logic controllers that avoid error.

A useful property of employing the satisficing principle in fuzzy logic controller design is the effect upon defuzzifier specification. Usually, the rationality behind defuzzification-a process which has been described as "an art rather than a science" [12]—is that of finding the best decision or control. This is typified not only by efforts to formulate the defuzzification problem as "the problem of optimal selection" [13, p. 38], but also by interpretations of fuzzy logic systems as universal function approximators [12]. For the cost/benefit-based satisficing fuzzy logic controller specified herein, defuzzifier design is motivated by the acceptability of multiple controls and, hence, enjoys a degree of flexibility that is useful for the design of robust controllers.

\section{B. Related Literature}

In this section, we briefly review related literature. A more extensive review can be found in the companion technical report [14]. Model predictive control (MPC), also known as moving horizon and receding horizon control, is a method for designing controllers that operate in nonlinear, constrained, and uncertain environments. Successes in application are supported by theoretical advances, such as the characterization and specification of sufficient conditions for stability [15]-[18], and by algorithms that are computationally efficient and ensure disturbance rejection through state feedback [19], [20]. In this paper, we significantly extend the results from [4], [7] to include considerations and contributions from fuzzy logic and to include the ability to deal with conflicting information or multiple experts.

Increasingly, fuzzy logic researchers are addressing the implicit compilation of steps 1)-3) into 4). For example, in both [21] and [22], explicit models are used to determine system behavior, which is then used to generate fuzzy controls via conventional fuzzy and classical control methods, respectively. An alternative to these approaches generates a fuzzy model and then employs stability as the sole performance criterion [23], [24]. In terms of Fig. 1, such approaches infer $c$ from $u$ and $\theta$ and then use $c$ to determine action $u$. These applications are primarily motivated by the desire to establish the provable stability of fuzzy logic controllers and to formulate systematic methods for controller design. Such approaches seek to combine objective mathematical knowledge with subjective fuzzy knowledge [25], [26]. In this paper, we combine the objective knowledge produced by mathematical models of nonlinear systems with a subjective interpretation of how local model predictions imply global system consequences. The use of local model predictions may free the expert from some of the burden of performing mental simulations of the plant [6] and instead allow the expert to identify consequences as beneficial or costly, even for unintuitive plants [21].

Satisficing presents a decision-making paradigm that differs from the de facto paradigm of optimality [27]-[30]. Many cognitive scientists recognize that insistence on optimality is a misplaced requirement in situations of limited resources and information and that optimality inadequately describes observed behavior in naturalistic settings [10], [31]-[33]. Additionally, the definition of and reliance upon an optimal solution has been questioned by Zadeh [5], [34] and other philosophers, scientists, and researchers concerned with pragmatic decision-making [2], [3], [35], [36]. The relationship between fuzzy logic-based satisficing and set-valued maximization is further explored in the companion paper [14].

Our treatment of uncertainty relies on higher order uncertainty [37] and, specifically, set-based Bayesianism [38]. Set-based Bayesianism permits a set of probabilities to describe uncertainty and under certain conditions subsumes Dempster-Shafer theory as a special case [39]. Rather than adopting a risk-averse stance such as minimax or a risk-neutral stance such as expectation, set-based Bayesianism allows an intermediate stance to be taken. This intermediate stance takes expectations with respect to a set of probabilities; a control is justified only if it is acceptable for each expected consequence. This is similar to requirements for robustness in set-theoretic estimation, including other developments of the satisficing concept [27], [28], [40]-[43].

\section{Strongly SATISFICING DeCision THEORY}

As discussed in the review of relevant literature, many cognitive scientists recognize that insistence on optimality is a misplaced requirement in situations of limited resources and information. Simon [9] addressed the issue of limited or bounded rationality by defining an aspiration level such that once this level is met the corresponding solution is deemed adequate or satisficing. ${ }^{1}$ An important characteristic of Simon's satisficing principle is that decisions are deemed adequate on the basis of a comparison: any decision which exceeds the aspiration level is admissible. We employ this characteristic by constructing and comparing two set membership functions similar to the way benefit and cost are compared in economics literature. This comparison leads naturally to a constructive procedure for identifying satisficing decisions in nonlinear

\footnotetext{
${ }^{1}$ A term employed by Simon. A convenient mnemonic is satisfice $=$ satisfy + suffice.
} 
system controller design contexts using receding planning horizons. The key to this development lies in partitioning the consequences into a generalized type of benefit called accuracy and a generalized type of cost called liability. These two decision attributes may be operationally characterized as follows.

Accuracy: A natural characterization of the benefit of a decision is accuracy, meaning conformity to a standard. In practical contexts, the standard corresponds to whatever goal or objective is relevant to the problem and accuracy corresponds to the degree of success in achieving that goal. In the context of fuzzy logic, the term accuracy refers to the set membership function associated with the linguistic variable ACCURATE. ${ }^{2}$

Liability: Actions may also be evaluated strictly in terms of their liability, meaning susceptibility or exposure to undesirable consequences. Typically, these consequences may be manifest in the form of costs or other penalties that would accrue simply as a result of taking the action, regardless of its accuracy. Liability corresponds to the degree to which actions accrue costs or penalties. In the context of fuzzy logic, the term liability refers to the set membership function associated with the linguistic variable LIABLE.

For example, in regulator design, the fundamental objective is to drive the system to and maintain the system at a desired operating point. Thus, accuracy refers to the degree to which the possible controlled states satisfy this objective. Independent of the desire to regulate the system is the desire to prevent excessive control authority and oscillatory state transitions. Thus, liability refers to the cost of possible controlled states with respect to these undesirable consequences.

Given these two evaluations of consequences, two independent principles can be applied: satisficing and domination. The satisficing principle (as we have used it) provides a mechanism for determining what action can be done given the observed evidence; the domination principle provides a mechanism for determine what actions should not be done given the alternative actions. For some problems such as constrained decision-making and task-based behavior [44], the satisficing principle can be applied without applying domination; and for other problems such as conventional multi-attribute decision analysis, the domination ${ }^{3}$ principle can be applied without satisficing.

\section{A. Satisficing Decisions}

Using Levi's error avoidance principle [11], SSDT provides a method by which the accuracy and liability set membership functions can be merged: to avoid error, a decision maker accepts those decisions which are ACCURATE and not LIABLE. Formally, let $U$ denote the set of possible decisions or actions and let $\Theta$ denote the states of nature. The states of nature represent those conditions, which affect the consequence of a decision but which cannot be controlled.

\footnotetext{
${ }^{2}$ For the remainder of the paper, we use capital letters and a separate font when we refer to linguistic variables, but will make no such distinction for membership functions.

${ }^{3}$ Because domination is an extension of the de facto optimality presumption, this principle is much more frequently encountered in decision making.
}

For each decision $u \in U$ and for each state of nature $\theta \in \Theta$, a consequence results ${ }^{4}$ that is the effect of making decision $u$ when nature is in state $\theta$. The accuracy $\mu_{A}: U \times \Theta \mapsto \mathbb{R}$ and liability $\mu_{L}: U \times \Theta \mapsto \mathbb{R}$ set membership functions are defined for each consequence (i.e., action/state-of-nature pair).

In SSDT, the set of all decisions which cannot be justifiably eliminated is called the satisficing set and is linguistically defined as

$$
\text { SATISFICING }=\text { ACCURATE } \text { and not(LIABLE). }
$$

For the problems addressed herein, we wish to include multiplicative hedges $\alpha, \rho \in[0,1]$, which allow the fuzziness inherent in the consequences of an action to be parameterized. Thus, we form the satisficing set membership function as

$$
\mu_{S_{b}}=\alpha \mu_{A} \star\left(1-\rho \mu_{L}\right)
$$

where $b$ represents a design parameter that is related to $\rho$ and $\alpha$, where $\star$ represents a $t$-norm, and where $1-\rho \mu_{L}$ represents the complement of the hedged set $\rho \mu_{L}$. When $\star$ represents the $t$-norm $Y \star Z=\max (0, Y+Z-1)$ (see, for example, [46]), the satisficing set membership becomes

$$
\begin{aligned}
\mu_{S_{b}} & =\max \left(0, \alpha \mu_{A}-\rho \mu_{L}\right)=\max \left(0, \mu_{A}-\frac{\rho}{\alpha} \mu_{R}\right) \\
& =\max \left(0, \mu_{A}-b \mu_{L}\right)
\end{aligned}
$$

where $b=\rho / \alpha \geq 0$ is called the rejectivity and parameterizes the relative weight ${ }^{5}$ between accuracy and liability.

The comparative nature of (2) is best illustrated by considering the region of support (area of nonzero set membership) for the satisficing set, which is given by

$$
S_{b}(\theta)=\left\{u: \mu_{A}(u ; \theta) \geq b \mu_{L}(u ; \theta)\right\}
$$

From (3) we see that decisions are satisficing if and only if the accuracy membership is large relative to the liability membership. This comparison provides a set-based mathematical formalism necessary to use the notion of satisficing in controller design. Through this comparison, each potential action may be evaluated on its own merits without comparing it to other actions. It is easily shown that for any membership functions defined such that $\int \mu_{A}(u ; \theta) d u=\int \mu_{L}(u ; \theta)=1$ or $\sup _{u} \mu_{A}(u ; \theta)=\sup _{u} \mu_{L}(u ; \theta)=1$, a sufficient condition for $S_{b}(\theta) \neq \emptyset$ is that $b<1$. In practice, this condition is overly conservative and is replaced by the operational restriction $b<\sup \left\{\beta \geq 0: S_{\beta} \neq \emptyset\right\}$. Note that since we restrict attention to membership functions such that the maximum of $\mu_{A}$ and $\mu_{R}$ is unity, the supremum exists and is finite.

\footnotetext{
${ }^{4} \mathrm{~A}$ decision $u$ is often treated as a mapping from $\Theta$ into the set of consequences [45].

${ }^{5}$ The subjective selection of this relative weighting is analogous to the tradeoff between the size and power of a statistical hypothesis test using Neyman-Pearson decision theory. Similar to the way in which subjectively selecting a test's size determines the test's power in Neyman-Pearson hypothesis testing, subjectively selecting $b$ determines the relative importance of accuracy and liability.
} 


\section{B. Strongly Satisficing Decisions}

Although the set $S_{b}$ contains all possible actions that are legitimate candidates for adoption, they generally will not be equal in overall quality. For example, two satisficing actions may have similar accuracy membership but have significantly different liability membership and implementing the one with the lower liability will yield essentially the same fuzzy benefit with lower fuzzy cost. Thus, we are motivated to refine the set of satisficing actions. For every $u \in U$ let

$$
\begin{gathered}
B_{A}(u ; \theta)=\left\{v \in U: \mu_{L}(v ; \theta)<\mu_{L}(u ; \theta)\right. \text { and } \\
\left.\mu_{A}(v ; \theta) \geq \mu_{A}(u ; \theta)\right\} \\
B_{L}(u ; \theta)=\left\{v \in U: \mu_{L}(v ; \theta) \leq \mu_{L}(u ; \theta)\right. \text { and } \\
\left.\mu_{A}(v ; \theta)>\mu_{A}(u ; \theta)\right\}
\end{gathered}
$$

and define the set of actions that are strictly better than $u$ (i.e., set of actions that dominate $u$ )

$$
B(u ; \theta)=B_{A}(u ; \theta) \cup B_{L}(u ; \theta)
$$

that is, $B(u ; \theta)$ consists of all possible actions that have lower liability but not lower accuracy than $u$ or have higher accuracy but not higher liability than $u$. If $B(u ; \theta)=\emptyset$, then no actions can be preferred to $u$ in both accuracy and liability and $u$ is a (weakly) nondominated action with respect to $\theta$. The (crisp) nondominated set

$$
\mathcal{E}(\theta)=\{u \in U: B(u ; \theta)=\emptyset\}
$$

contains all nondominated actions.

The intersection of the nondominated set with the satisficing set yields the strongly satisficing set

$$
\begin{aligned}
\mathcal{S}_{b}(\theta) & =\mathcal{E}(\theta) \cap S_{b}(\theta) \\
\mu_{\mathcal{S}_{b}} & = \begin{cases}\mu_{S_{b}}, & u \in \mathcal{E}(\theta) \\
0, & \text { otherwise }\end{cases}
\end{aligned}
$$

where $\mathcal{S}_{b}(\theta)$ represents the crisp support set given $\theta$. When $S_{b}$ is nonempty, it has been shown that $\mathcal{S}_{b}(\theta)$ is also nonempty [7]. Intuitively, $S_{b}(\theta)$ contains only those controls which can be done given the evidence and $\mathcal{E}$ does not contain any controls which should not be done given the alternatives. Elements of $\mathcal{S}_{b}$ exhibit both properties where, as we shall demonstrate, the strongly satisficing set can facilitate flexible defuzzifier design.

\section{Robustness}

The accuracy and liability set membership functions are defined such that both $\mu_{A}(u ; \cdot)$ and $\mu_{L}(u ; \cdot)$ are measurable functions defined over $\Theta$ for fixed $u$ and both $\mu_{A}(\cdot ; \theta)$ and $\mu_{L}(\cdot ; \theta)$ are set membership functions for fixed $\theta$. These membership functions represent the value of a decision $u$ when the state of nature is $\theta$ or stated simply, represent the statement if $\theta$ then $u$ is ACCURATE/LIABLE. By contrast inferring a decision directly from the observations (if $\theta$ then $d o u$ ), we instead infer the value of a decision from the observations (if $\theta$ then $u$ is (not) valuable). When the state of nature is $\theta^{*}$ instead of $\theta$ or when $\theta$ is nonsingleton with membership $f_{\theta}(\theta)$, then the accuracy/liability of $u$ is obtained by forming the composition of the linguistic statements $\theta^{*}$ and if $\theta$ then $u$ is-. For the applications in this paper, the composition is defined by taking the expected accuracy/liability membership with respect to the state of nature described by the membership function $f_{\theta}$ (which is constrained to be a probability density function). Note that other compositions can also be considered.

For some problems, there may exist multiple descriptions of $\theta$ and it is desirable to include each of these multiple descriptions in the definition of $S_{b}$. For example, there may be two experts who specify accuracy and liability membership functions; i.e., there exist $\Theta_{1}$ and $\Theta_{2}$ and corresponding membership functions $\mu_{A}\left(u ; \theta_{\gamma}\right), \mu_{L}\left(u ; \theta_{\gamma}\right)$, and $f_{\theta}^{\gamma}(\theta), \gamma \in$ $\{1,2\}$. For the applications presented herein, ${ }^{6}$ we restrict attention to expert/system descriptions that share a common state of nature $\Theta$ and common evaluations of consequences $\mu_{A}(u ; \theta), \mu_{L}(u ; \theta)$, but differ in their descriptions of nature $f_{\theta}^{\gamma}$. Formally, let

$$
\mathcal{F}=\left\{f_{\theta}^{\gamma}: \Theta \mapsto \mathbb{R} ; \gamma \in \Gamma\right\}
$$

denote a closed set of subjective probability densities that represent a designer's understanding of the state of nature, where the set $\Gamma$ represents an index of this set (for example, $\Gamma=\{1,2, \cdots\})$. For simplicity, attention is restricted to countable $\Gamma$. Corresponding to each $f_{\theta}^{\gamma} \in \mathcal{F}$ is an expected accuracy $\bar{\mu}_{A}^{\gamma}$ and an expected liability $\bar{\mu}_{L}^{\gamma}$ given by

$$
\begin{aligned}
& \bar{\mu}_{A}^{\gamma}(u)=\int_{\Theta} \mu_{A}(u ; \theta) f_{\theta}^{\gamma}(\theta) d \theta, \\
& \bar{\mu}_{L}^{\gamma}(u)=\int_{\Theta} \mu_{L}(u ; \theta) f_{\theta}^{\gamma}(\theta) d \theta .
\end{aligned}
$$

When $\Gamma$ contains only one element, the definitions for the satisficing set $S_{b}^{\gamma}$ and nondominated set $\mathcal{E}^{\gamma}$ in (2)-(3) and (6), respectively, are modified by using $\bar{\mu}_{A}^{\gamma}(u)$ and $\bar{\mu}_{L}^{\gamma}(u)$ instead of $\mu_{A}(u ; \theta)$ and $\mu_{L}(u ; \theta)$. By contrast, when $\Gamma$ contains more than one element (i.e., there are more than one measurement source or expert opinion), a decision $u \in U$ is satisficing if and only if it is satisficing for all $f_{\theta}^{\gamma} \in \mathcal{F}$. This is similar to requirements for robustness in set-theoretic estimation, including other developments of the satisficing concept [27], [28], [40]. The resulting set is called the robust satisficing set and is defined as

$$
\begin{aligned}
S_{b} & =\left\{u: \text { for all } \gamma \in \Gamma, \bar{\mu}_{A}^{\gamma}(u) \geq b \bar{\mu}_{L}^{\gamma}(u)\right\} \\
& =\bigcap_{\gamma \in \Gamma} S_{b}^{\gamma} \\
\mu_{S_{b}} & =\min _{\gamma \in \Gamma} \mu_{S_{b}^{\gamma}} .
\end{aligned}
$$

One criticism against using the intersection operator to fuse results generated by multiple sources is that the resulting set may be empty. However, the design parameter $b$ can be selected such that the robust satisficing set is always nonempty. This is achieved if and only if $b$ is bounded by $b<\inf _{\gamma \in \Gamma} b^{\gamma}$, where $b^{\gamma}=\sup \left\{b \geq 0: S_{b}^{\gamma} \neq \emptyset\right\}$. Loosely speaking, $b$ is bounded by the minimax value over all nonvacuous $\left(S_{b} \neq \emptyset\right)$ expert opinions.

\footnotetext{
${ }^{6}$ Using the more general framework of multiple states of nature $\Theta_{i}$, we establish a foundation for designing controllers which fuse multiple sources of sensor information.
} 
The robust nondominated set can also be defined by eliminating controls for which obviously better (dominating) controls exist. Let

$$
\begin{aligned}
& B_{A}^{\gamma}(u)=\left\{v \in U: \bar{\mu}_{L}^{\gamma}(v)<\bar{\mu}_{L}^{\gamma}(u) \text { and } \bar{\mu}_{A}^{\gamma}(v) \geq \bar{\mu}_{A}^{\gamma}(u)\right\} \\
& B_{L}^{\gamma}(u)=\left\{v \in U: \bar{\mu}_{L}^{\gamma}(v) \leq \bar{\mu}_{L}^{\gamma}(u) \text { and } \bar{\mu}_{A}^{\gamma}(v)>\bar{\mu}_{A}^{\gamma}(u)\right\} \\
& B^{\gamma}(u)=B_{A}^{\gamma}(u) \cup B_{L}^{\gamma}(u) .
\end{aligned}
$$

Then

$$
\mathcal{E}=\left\{u: \bigcap_{\gamma \in \Gamma} B^{\gamma}(u)=\emptyset\right\} .
$$

In general, this set can be difficult to compute. Since, as the following theorem shows, $\bigcup_{\gamma \in \Gamma} \mathcal{E}^{\gamma} \subseteq \mathcal{E}$, we can use the following approximation for the robust equilibrium set:

$$
\tilde{\mathcal{E}}=\bigcup_{\gamma \in \Gamma} \mathcal{E}^{\gamma}
$$

Theorem 1: $\bigcup_{\gamma \in \Gamma} \mathcal{E}^{\gamma} \subseteq \mathcal{E}$.

Proof: Suppose $u \in \cup_{\gamma \in \Gamma} \mathcal{E}^{\gamma}$. Then there exists a $\gamma_{i}$ such that $u \in \mathcal{E}^{\gamma_{i}}$ where $B^{\gamma_{i}}(u)=\emptyset$. Since $B^{\gamma_{i}}(u)=\emptyset$ it follows that $\cap_{\gamma \in \Gamma} B^{\gamma}(u)=\emptyset$ where $u \in \mathcal{E}$.

Since each $\mathcal{E}^{\gamma}$ is always nonempty, $\tilde{\mathcal{E}}$ is also always nonempty. For the applications considered in this paper, $\mathcal{E}=\tilde{\mathcal{E}}$ so we use the more simple notation.

The robust strongly satisficing set can now be defined by restricting the robust satisficing set $S_{b}$ to the region of support defined by the robust equilibrium set $\mathcal{E}$. The robust satisficing set can be expressed as

$$
\begin{aligned}
\mathcal{S}_{b} & =\mathcal{E} \cap S_{b}=\left(\underset{\gamma \in \Gamma}{\cup} \mathcal{E}^{\gamma}\right) \cap\left(\underset{\gamma \in \Gamma}{\cap} S_{b}^{\gamma}\right) \\
\mu_{\mathcal{S}_{b}} & = \begin{cases}\mu_{S_{b}}, & u \in \mathcal{E} \\
0, & \text { otherwise }\end{cases}
\end{aligned}
$$

from which we see that (7) and (8) are special cases with $\Gamma$ a singleton and $f_{\theta}$ a delta function placing all belief mass on the single value $\theta$. We now have a general definition for a robust strongly satisficing control. Broadly speaking, a decision is robustly strongly satisficing if and only if it is satisficing for every belief held by the designer and nondominated for any such belief. Thus, if a designer is unsure of precisely how nature should be fuzzified ${ }^{7}$ but can restrict the description to within a set, then the designer can choose a control which is justifiable for all descriptions.

In the following theorem, we present a sufficient (but not necessary) condition to guarantee that the robust strongly satisficing set is nonempty. The interpretation of this theorem is as follows: if there is a decision such that $\left(\cap_{\gamma \in \Gamma} \bar{\mu}_{A}^{\gamma}\right.$ and not $\cup_{\gamma \in \Gamma} \bar{\mu}_{L}^{\gamma}$ ) has nonzero support, then the robust strongly satisficing set has nonzero support. In words, if $u$ is ACCURATE for every fuzzified state and not LIABLE for any fuzzified state, then $u$ is strongly satisficing.

Theorem 2: If there exists a $u \in U$ such that $\min _{\gamma \in \Gamma} \bar{\mu}_{A}^{\gamma}(u) \geq b \max _{\gamma \in \Gamma} \bar{\mu}_{L}^{\gamma}(u)$ then $\mathcal{S}_{b} \neq \emptyset$.

\footnotetext{
${ }^{7}$ In our usage, fuzzification is used in the sense of [47].
}

Proof: We will prove that $\mathcal{S}_{b}$ is nonempty for these conditions by constructing a specific element of this set. Let

$$
u_{D}=\arg \max _{u \in U}\left\{\min _{\gamma \in \Gamma}\left\{\bar{\mu}_{A}^{\gamma}(u)\right\}-b \max _{\gamma \in \Gamma}\left\{\bar{\mu}_{L}^{\gamma}(u)\right\}\right\}
$$

denote the (robustly) most discriminating control. We first show that $u_{D} \in S_{b}$. Since $\min _{\gamma \in \Gamma} \bar{\mu}_{A}^{\gamma}\left(u_{D}\right) \geq$ $\max _{\gamma \in \Gamma} \bar{\mu}_{L}^{\gamma}\left(u_{D}\right)$ (otherwise it is not most discriminating given the hypothesis), then for any $\gamma \in \Gamma, \bar{\mu}_{A}^{\gamma}\left(u_{D}\right) \geq$ $\min _{\rho \in \Gamma} \bar{\mu}_{A}^{\rho}\left(u_{D}\right) \geq \max _{\rho \in \Gamma} \bar{\mu}_{L}^{\rho}\left(u_{D}\right) \geq \bar{\mu}_{L}^{\gamma}\left(u_{D}\right)$ implies that $u_{D} \in S_{b}^{\gamma}$, where $u_{D} \in S_{b}$.

We now show ${ }^{8}$ that $u_{D} \in \mathcal{E}$. For any $\gamma \in \Gamma$, suppose that $u_{D} \notin \mathcal{E}^{\gamma}$. Then there is a $u \in B_{A}^{\gamma}\left(u_{D}\right) \cup B_{L}^{\gamma}\left(u_{D}\right)$ where

$$
\begin{aligned}
\bar{\mu}_{A}^{\gamma}(u)-b \bar{\mu}_{L}^{\gamma}(u) & >\bar{\mu}_{A}^{\gamma}\left(u_{D}\right)-b \bar{\mu}_{L}^{\gamma}\left(u_{D}\right) \\
& \geq \min _{\gamma \in \Gamma} \bar{\mu}_{A}^{\gamma}\left(u_{D}\right)-b \max _{\gamma \in \Gamma} \bar{\mu}_{L}^{\gamma}\left(u_{D}\right)
\end{aligned}
$$

which means that $u$ is most discriminating. This contradicts our assumption where $u_{D} \in \mathcal{E}^{\gamma}$ for all $\gamma \in \Gamma$, where $u_{D} \in \mathcal{E}$.

Since $u_{D} \in \mathcal{E}$ and $u_{D} \in S_{b}$ then $u_{D} \in \mathcal{S}_{b}$, where $\mathcal{S}_{b} \neq \emptyset$.

Note that the operator $\min _{\gamma \in \Gamma} \bar{\mu}_{A}^{\gamma}$ de-emphasizes the good and the operator $\max _{\gamma \in \Gamma} \bar{\mu}_{L}^{\gamma}$ overemphasizes the bad yielding a conservative controller.

\section{Defuzzifier Design}

Defuzzifiers for typical fuzzy logic systems employ superlative methods such as the maximum defuzzifier or averaging methods such as the centroid defuzzifier [12]. In these methods, a single control is selected from the set of controls with nonzero set membership because it is superior or most representative. Selection and design of a defuzzifier can be a significant obstacle in designing a fuzzy logic controller [12]. We suggest that one reason defuzzifier design is difficult is that many rule bases are based upon local rather than than global performance. A well-known result from optimal control theory is that optimizing over a local planning horizon does not necessarily yield global optimal performance. Thus, we suggest that rule bases determined by local performance considerations may be incompatible with optimal defuzzifying and may instead require "procedurally rational" defuzzifying [4].

The satisficing fuzzy logic controller (SFLC) includes both the accuracy set membership function as well as the liability set membership function. Unlike most fuzzy control applications (but similar to the ART model [48, p. 106]), the output vector of the SFLC does not correspond to a final control actions; i.e., inference is not made from the observation to a control solution. Instead, inference from the observation to the control solution proceeds in three distinct steps: 1) observe nature and infer consequences of control actions through an explicit dynamic model; 2) infer values from the consequences using an explicit expert; and 3) infer the fuzzy set control solutions from values using both the satisficing and domination principles. Note that when $b=0$ and when the accuracy inference is made directly from observations to values then

\footnotetext{
${ }^{8}$ Note that we actually show the stronger result that $u_{D} \in \cap_{\gamma \in \Gamma} \mathcal{E}^{\gamma}$.
} 
the conventional FLC is obtained and, thus, the SFLC can be viewed as a generalization of the FLC.

For the SFLC, any defuzzifier which selects a control from $\mathcal{S}_{b}$ is justifiable according to two criteria: 1) all such controls are satisficing (in $S_{b}$ ), which means that evidence is sufficient to justify their use and 2) all such controls are maximizing [in (1), $\mathcal{E}=\mathfrak{E}$, see (16)] for some $b$, which means that no other control is superior and thereby precludes their use. Furthermore, when $\mathcal{F}$ (the set of beliefs held by the designer) is a singleton, then the set of strongly satisficing controls can claim superiority in the following sense and, hence, can be justified as the defuzzifier output. Let

$\mathfrak{E}=\left\{u: \exists b^{u}>0\right.$ for which $\left.u=\arg \max _{v \in U} \bar{\mu}_{A}(v)-b \bar{\mu}_{L}(v)\right\}$.

This set consists of those actions $u$ which maximize the difference between the accuracy and liability set membership functions for some value of $b$. A well-known result from multiobjective optimization, which is presented in the companion report [14] in this context for completeness and for a specific case in [7], implies that the set $E=\mathcal{E}$. This means that nondominated solutions are not only superior to all other solutions in some sense, they are also mathematically optimizing.

\section{Model Predictive SATISFICING FUZZY LOGIC CONTROLLER SYNTHESIS}

It is desirable to employ expert knowledge to determine how local information translates into global goal achievement. For example, automobile drivers are (usually) very effective at interpreting how local measurements (such as time headway and relative velocity) translate into global consequences (such as safe but expedient travel) [44]. Such a translation between local measurements and decisions reflecting global consequences must either implicitly or explicitly address the inferences diagrammed in Fig. 1. In this section, we develop methods for implementing such inferences and identify restrictions that facilitate computable methods for performing such inferences. We first briefly review how consequences can be inferred from observations using receding horizon model predictions [7]. We then discuss how these predictions can be used to infer valuations of control. Finally, we review how these valuations can be used to infer justifiable behavior.

\section{A. Consequences: Receding Planning}

\section{Horizons and Influence Vectors}

Consider a discrete time, time-varying single input nonlinear plant of the form

$$
\boldsymbol{x}(t+1)=f_{1}[\boldsymbol{x}(t), t]+\boldsymbol{f}_{2}[\boldsymbol{x}(t), t] u(t)+\boldsymbol{g}[\boldsymbol{x}(t), t] v(t)
$$

where $\boldsymbol{x}(t)$ represents the system state, $u(t)$ is the system input, and $v(t)$ is a disturbance. Given a desired state $\boldsymbol{x}^{*}=0$ and positive definite cost matrices $P, Q$, and $R$, the conventional model predictive controller (see, for example, [17] and [18]) is obtained by minimizing

$$
\begin{aligned}
J_{N}= & \boldsymbol{x}(t+N)^{T} P \boldsymbol{x}(t+N)+\sum_{k=0}^{N-1}\left[u(t+k)^{T} R u(t+k)\right. \\
& \left.+\boldsymbol{x}(t+k+1)^{T} Q \boldsymbol{x}(t+k+1)\right]
\end{aligned}
$$

with respect to the control sequence $u(t), \cdots, u(t+N-$ 1) subject to the control bounds and dynamics constraint given by, respectively [17], $u(t+k) \in U$ for all $k$ and $\Delta u(t+k) \in \Delta U$ for all $k$. The first control $u(t)$ of the resulting minimizing sequence is applied and the constrained minimization is repeated for the next and all subsequent time steps. Although for some model predictive systems there exist methods for guaranteeing plant stability (such as including a terminal constraint), the cost matrices $P, Q$, and $R$ are specified by an implicit expert (the designer) to infer how predicted states translate into global plant performance.

In [7], we developed the notion of an influence vector $\chi(u)$ defined for the discrete time dynamical system in (17) and then use this influence vector and the standard quadratic cost function in (18) to develop a receding horizon controller. Let $\boldsymbol{\chi}_{\ell}(t+\tau)$ be the substate of $\boldsymbol{x}(t+\tau)$ that is an explicit function of $u(t+\ell), \tau=1, \cdots, D_{t+\ell}$, where $D_{t+\ell}$ is the maximum number of time increments for which $u(t+\ell)$ has explicit influence on any component of the state. For a planning horizon $N$, the influence vectors are the $N$ sequences of substates of the form

$$
\begin{aligned}
\chi_{0}(u(t))= & \left\{\boldsymbol{\chi}_{0}(t+1), \cdots, \boldsymbol{\chi}_{0}\left(t+D_{t}\right)\right\} \\
\chi_{1}(u(t+1))= & \left\{\boldsymbol{\chi}_{1}(t+2), \cdots, \boldsymbol{\chi}_{1}\left(t+1+D_{t+1}\right)\right\} \\
\boldsymbol{\chi}_{N-1}(u(t+N-1))= & \left\{\boldsymbol{\chi}_{N-1}(t+N)\right. \\
& \left.\cdots, \boldsymbol{\chi}_{N-1}\left(t+N-1+D_{t+N-1}\right)\right\} .
\end{aligned}
$$

By restricting attention to single step receding horizon, $u(t)$ is the only relevant control variable where we can write $\boldsymbol{\chi}(t)=\chi_{0}(t)$. For the plant in (17), it can be shown (see [7]) that the influence vector can be written in the form

$$
\boldsymbol{\chi}(t)=\left[x_{1}\left(t+\delta_{1}\right), \cdots, x_{n}\left(t+\delta_{n}\right)\right]^{T}
$$

where $\boldsymbol{x}(t)=\left[x_{1}(t), \cdots, x_{n}(t)\right]^{T}$, and where $\delta_{i}$ is the delay before $u(t)$ influences the ith element of the vector $\boldsymbol{x}$. A necessary restriction ${ }^{9}$ is that each $x(t)$ is controllable via $u(t)$; that is, each $\delta_{i}$ is defined and there exists and $N$ such that for any $x(0)$ there exists a sequence of controls $u(t), \cdots$, $u(t+N-1)$ such that $\boldsymbol{x}(N)=\mathbf{0}$. Given these restrictions, $D_{t}=\max _{i} \delta_{i}$. Using the procedure in [7], each $\delta_{i}$ is easily computed subject to the plant in (17). As in [7], we require that although all system matrices can be time-varying, the variations are restricted such that each $\delta_{i}$ is constant.

\section{B. Observations, Consequences, and Evaluations}

Since modeling is subject to uncertainty $\left(f_{1}, f_{2}\right.$, and $g$ in (17) may not be precisely known) it is desirable to develop controllers that work for multiple system models. It is also desirable to develop controllers that operate effectively in

\footnotetext{
${ }^{9} \mathrm{An}$ area of current active research is the development of stable satisficing controllers. A key to this development is the controllability of the plant.
} 
the presence of external disturbances $v$. This is one of the objectives of robust control design (see, for example, [41], [42], [43]). In this paper, ${ }^{10}$ we restrict attention to problems for which precise measurements of $\boldsymbol{x}$ are available, thereby focusing emphasis on robustness with respect to nonwhite disturbances as well as with respect to model uncertainty. Thus, $\theta=\left(f_{1}, f_{2}, g, v\right)$. An estimate $\hat{\theta}$ and its associated distribution $f_{\theta}$ then gives us information necessary to make a decision. When disturbances are difficult to predict it is helpful to consider a set of such predictions in determining a control. Similarly, when system models are subject to error, it is helpful to consider a set of such models. Thus, if more than one disturbance/system model ${ }^{11}$ is possible, then the designer can consider more than one $f_{\theta}(\theta)$ in determining a control $u$.

In the cost function given by (18), there are two different factors: the terminal cost $\boldsymbol{x}^{T} P \boldsymbol{x}$ and the "cost to go" $\boldsymbol{x}^{T} Q \boldsymbol{x}+$ $u^{T} R u$. Rather than aggregating the terminal cost and "cost to go" into a single global performance metric, we can instead independently identify and compare each factor from a local perspective [7]. Thus, we define the accuracy cost functional for a single step control horizon as the terminal cost portion of the receding horizon cost function

$$
\Phi(u ; \theta)=\chi^{T}(t) P \boldsymbol{\chi}(t)
$$

and a liability cost functional for a single step control horizon as the "cost-to-go" portion of the receding horizon cost function

$$
\Lambda(u ; \theta)=\chi^{T}(t) Q \chi(t)+u^{T}(t) R u(t)
$$

The accuracy cost functional is associated with the global goal of the controller and the liability cost functional is associated with the proximate design considerations. In [7] the matrices $P, Q$, and $R$ were assumed given by an implicit expert. In this paper, we specify these matrices using an explicit rule base obtained from expert knowledge. In words, the plant model specifies the inference from observations to consequences and the explicit expert specifies the relation between local (receding horizon) consequences and global (plant behavior) values.

\section{Decisions}

By normalizing (19)-(20), $\mu_{A}$ and $\mu_{L}$ may be determined as

$$
\begin{aligned}
& \mu_{A}(u ; \theta)=\kappa_{A}\left[\max _{z \in U}\{\Phi(z ; \theta)\}-\Phi(u ; \theta)\right] \\
& \mu_{L}(u ; \theta)=\kappa_{L}\left[\Lambda(u ; \theta)-\min _{z \in U}\{\Lambda(z ; \theta)\}\right]
\end{aligned}
$$

where $\kappa_{A}$ and $\kappa_{L}$ are the (possibly state dependent) normalizing constants required to create membership functions. Observe that since $\mathcal{E}(\theta)$ can be directly computed from (19)-(20), normalization can occur so that all mass is restricted to

\footnotetext{
${ }^{10}$ Following the example of [47], we could treat measurement uncertainty using fuzzified observations $\hat{\boldsymbol{x}}$. Thus, the state of nature could be extended to include $\theta=\left(\boldsymbol{x}, \boldsymbol{y}, \boldsymbol{f}_{1}, \boldsymbol{f}_{2}, \boldsymbol{g}, \boldsymbol{h}, v, w\right)$, where $\boldsymbol{y}=\boldsymbol{h}(\boldsymbol{x}(t), t)+w(t)$.

${ }^{11}$ Although criteria such as Akaike's Information Theoretic Criterion exist, it may be difficult for a designer to justify a model class as being the best class for a problem [49].
}

the appropriate region of support $\mathcal{E}$. Furthermore, observe that this normalization yields set membership functions that situationally depend on the set of available controls where fuzzy values are situationally inferred.

It is worth noting that the most discriminating control $u_{D}$ defined in (15) closely resembles the solution obtained by minimizing $\alpha \Phi+\beta \Lambda$ for $\alpha, \beta \geq 0$. As observed in [7], $u_{D}$ depends on a state-dependent evaluation of predicted consequences $\chi$ on the basis of what can be done (the control $u$ ) given the circumstances (the observation $\theta$ ). By contrast, minimizing $\alpha \Phi+\beta \Lambda$ reduces to a nonlinear state feedback control law that does not explicitly consider the set of available alternatives. Additionally, as demonstrated in Section IV-B2 for plants with no known feedback law in closed form, a search to find $u_{D}$ need not be performed each sample time whereas a search for the minimizing solution of $\alpha \Phi+\beta \Lambda$ must be performed for each sample time.

\section{EXAMPLES}

In this section, we develop rule bases and present results for the rotational translation actuator (RTAC) [50], [51] and the inverted pendulum problems. These problems are appropriate because they serve as benchmarks for demonstrating "proof of concept." In the examples, not only is the set-based structure of SSDT used to provide robustness to nonwhite disturbances and model uncertainty, but also the resulting flexibility in defuzzifier design is also illustrated.

In the preceding sections, we have taken care to discriminate between three types of inference: 1) inferring (partial) consequences from observations; 2) inferring valuations from (partial) consequences; and 3) inferring decisions from valuations. These inferences were resolved in Section III by 1) one-step predictions of consequences obtained using an explicit plant model; 2) the terminal and cost-to-go performance metrics defined by an expert and subject to facilitatory restrictions; and 3) the strongly satisficing decision theory described in Section II. In this section, we focus on implementing these inferences in examples, and illustrate some of the implications.

Special mention needs to be made of the process of inferring valuations from consequences. As implemented, an expert must not only specify a rule base structure to determine cost functions, but also specify numerical parameters for these cost functions. This rule base structure and numerical specification are required during design-time but not employed during execution time. We present rule bases for two problems and assume that an expert exists who can translate the resulting structured cost-functions into numerical representations. In practice, such translation was accomplished by iteratively adjusting these parameters in simulation. Note that, because the specified cost functions are normalized to produce setmembership functions and thereby ensure the comparability of accuracy and liability, the expert need only specify relative numbers (ratios) of the usefulness of each rule. This process is compatible with a "tune by simulations" approach.

\section{A. The RTAC}

The RTAC system is shown in Fig. 2 and represents a translational oscillator with an eccentric rotational proof mass 


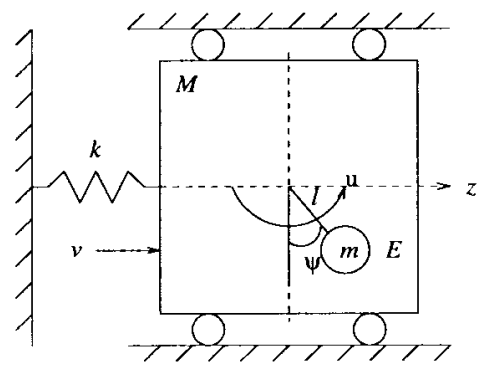

Fig. 2. Translational oscillator with rotational actuator.

connected by a linear spring of stiffness $k$ to a fixed wall. The cart is constrained to have one-dimensional travel. The rotational proof mass actuator affixed to the cart has mass $m$ and moment of inertia $E$ about its center of mass. Its center of mass is located a distance $l$ from the axis about which it rotates. It is assumed that motion occurs in a horizontal plane so that there are no gravitational forces to be considered. The control $u$ denotes a torque applied to the proof mass and $v$ denotes a disturbance force on the cart. The equations of motion are given by

$$
\begin{aligned}
(M+m) \ddot{z}+k z & =-m e\left(\ddot{\psi} \cos \psi-\dot{\psi}^{2} \sin \psi\right)+u \\
\left(E+m l^{2}\right) \ddot{\psi} & =-m l z \cos \psi+v
\end{aligned}
$$

which can easily be written in the form of (17) with $y=$ $\boldsymbol{x}=[\psi, x, \dot{\psi}, \dot{x}]^{T}$ and satisfies the conditions specified in Section III. The states $z$ and $\dot{z}$ denote the translational position and velocity of the cart, respectively, and $\psi$ and $\dot{\psi}$ denote the angular position and velocity of the rotational proof mass, respectively. In the simulation results, we use Euler integration with sample time $T=0.002 \mathrm{~s}$, and the following parameter values: $M=1.856 \mathrm{~kg} ; m=0.065 \mathrm{~kg} ; l=0.062 \mathrm{~m}$; $E=0.000014 \mathrm{~kg} \mathrm{~m}^{2}$; and (nominally) $k=203.7 \mathrm{~N} / \mathrm{m}$.

Although the discretized sub-states $\psi(t+1)$ and $z(t+1)$ are not explicit functions of $u(t)$, the substates $\dot{\psi}(t+1)$, $\dot{z}(t+1), \psi(t+2)$, and $z(t+2)$ are. Thus, we may identify the components of the state that are influenced (due to the relative order of the system) by the current input $u(t)$ as the velocity vector $\boldsymbol{v}(t+1)=[\dot{\psi}(t+1), \dot{z}(t+1)]^{T}$ and position vector $\boldsymbol{p}(t+2)=[\psi(t+2), z(t+2)]^{T}$ yielding $\boldsymbol{\chi}(t)=[\boldsymbol{p}(t+2), \boldsymbol{v}(t+1)]^{T}$.

1) Specification of Attributes: We now turn attention to determining a structure for $P, Q$, and $R$, and selecting $\kappa_{A}$ and $\kappa_{L}$ in (19)-(22) to yield desirable results. We normalize the membership functions such that they have unit area over the region of support $\mathcal{E}$. The corresponding normalizing constants are given by

$$
\begin{aligned}
\kappa_{A} & =\frac{1}{\int_{v \in \mathcal{E}}\left(\max _{z \in \mathcal{E}} \Phi(z)-\Phi(v)\right) d v} \\
\kappa_{L}= & \frac{1}{\int_{v \in \mathcal{E}}\left(\Lambda(v)-\min _{z \in \mathcal{E}} \Lambda(z)\right) d v} .
\end{aligned}
$$

For the simulation results presented herein, the robustly most discriminating defuzzifier defined in Theorem 2 is used.
The structures of the cost matrices and, hence, the values inferred from the set of consequences are determined by associating elements of these matrices with particular rules as follows. This problem is a regulator problem with the objective of keeping the cart close to the origin. Since regulation (a global consequence) cannot be precisely determined ${ }^{12}$ by considering only a single-step control horizon $(N=1)$, the set of accurate controls is fuzzy and, consequently, associated with a set-membership function. The accuracy of a control is dictated by the following rule: those controls for which $\chi$ is close to the origin (a consequence similar to regulation) have high accuracy. Since each element of $\chi$ is equally important with respect to this purpose, we let $P=I$.

Liability is determined by a set of rules obtained from expert-specified design principles and tuned by simulation. The design principles dictate the following rules for generating the liability membership function.

$A$ If cart position, proof mass angle, or velocities produced by a control are relatively large the control is liable.

$B$ If the cart position and cart velocity have the same sign the control is liable. This reflects expert understanding that when the cart is moving away from the origin the system is not very close to being in equilibrium.

$C$ If a control requires a great deal of energy it is liable.

These rules dictate a block diagonal structure of the matrix $Q$. We identify the relationships between elements of $\boldsymbol{\chi}$ dictated by the rules by placing the appropriate rule $(\mathrm{A}, \mathrm{B}, \mathrm{C})$ in the matrices below. If no relationship exists, then the matrix element is zero

$$
Q=\left[\begin{array}{cccc}
A & 0 & 0 & 0 \\
0 & A & 0 & B \\
0 & 0 & A & 0 \\
0 & B & 0 & A
\end{array}\right] \quad R=C .
$$

Given this rule-base structure, numerical values are tuned in simulation. Such tuning is tantamount to determining the relative importance of these local rules in relation to the global performance of the controller. After tuning, $Q$ becomes

$$
Q=\left[\begin{array}{cccc}
5 & 0 & 0 & 0 \\
0 & 5 & 0 & 30 \\
0 & 0 & 1 & 0 \\
0 & 30 & 0 & 5
\end{array}\right] \quad R=0.5
$$

Since rejecting disturbances by damping unfavorable cart motion is the most important factor for this system, the second rule gets the most emphasis (weight $B=30$ ). Note that $Q$ is not positive semi-definite; this formulation is permissible because we treat the quadratic forms as fuzzy inference engines rather than as cost functions used to produce the optimal behavior.

Note that $P, Q$, and $R$ are specified by experts at design time and are used to infer valuations from a partial understanding of global consequences. Run-time inferences to action are done using the resulting valuations via satisficing, domination, and defuzzifying. Also note that normalizing the resulting cost functions to give set membership functions yields valuations

\footnotetext{
${ }^{12}$ For example, nonminimum phase systems exhibit unintuitive behaviors for single-step control horizons [21].
} 


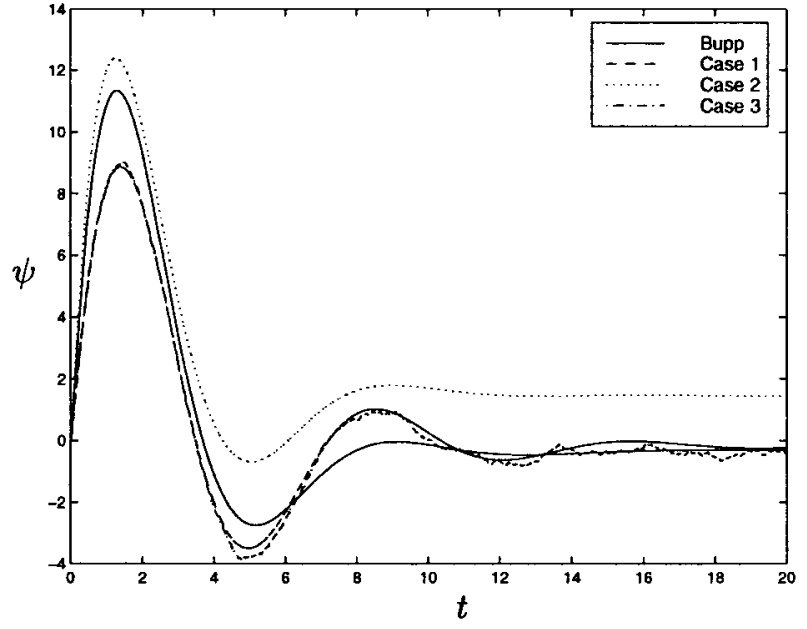

(a)

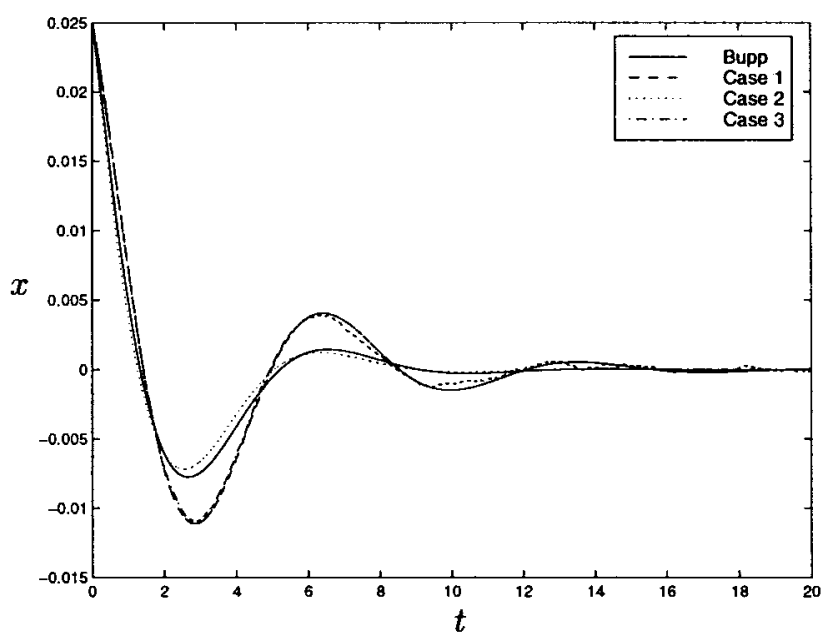

(b)

Fig. 3. Phase planes for the RTAC controllers. (a) Rotational-proof mass phase angle (in degrees). (b) Translational cart position (in meters).

that situationally depend on the set of available controls. Thus, values are situationally inferred.

2) Results: In the simulations, we consider three cases. The first case has known system parameters ( $\boldsymbol{f}$ and $\boldsymbol{g}$ from (17) are known) and is disturbance free $(v(t)$ from (17) is zero) where $\theta=(\boldsymbol{f}, \boldsymbol{g}, 0)$ is known precisely. The second case has no disturbances $(v(t)=0)$ but does have uncertain spring constant ( $\boldsymbol{f}$ uncertain, but $\boldsymbol{g}$ known) where $\theta^{\gamma}=$ $\left(f^{\gamma}, \boldsymbol{g}, 0\right)$. The third case has known system parameters $(\boldsymbol{f}$ and $\boldsymbol{g}$ are known) but has nonwhite disturbances generated by an unknown model structure where $\theta^{\gamma}=\left(\boldsymbol{f}, \boldsymbol{g}, v^{\gamma}\right)$. The time histories of the rotational-proof mass angle and the cart position obtained using a SFLC for each case are shown in Fig. 3(a) and (b), respectively. For qualitative comparison, simulation results using a discretized version of the stabilizing controller developed by Bupp et al., in [50] are also shown. For quantitative comparison, we define the observed cost function based on (18) for a $20 \mathrm{~s}$ simulation duration as

$$
\begin{aligned}
\tilde{J}_{t=20}= & \boldsymbol{x}(20)^{T} P \boldsymbol{x}(20)+\sum_{k=0}^{20 / T-1}\left[\boldsymbol{x}(k T+1)^{T} Q \boldsymbol{x}(k T+1)\right. \\
& \left.+u(k T)^{T} R u(k T)\right] .
\end{aligned}
$$

For case one, $\theta=(\boldsymbol{f}, \boldsymbol{g}, 0)$. The time histories of the rotational-proof mass angle and the cart position in Fig. 3 are for a satisficing controller with $b^{\text {case } 1}=3$. The satisficing controller behaves qualitatively (as shown in the plots) and quantitatively $\left(\tilde{J}_{t=20}^{\text {Bupp }}=219.6\right.$ and $\left.\tilde{J}_{t=20}^{\text {case } 1}=157.9\right)$ similar to Bupp's controller. ${ }^{13}$

For case two $\theta^{\gamma}=\left(\boldsymbol{f}^{\gamma}, \boldsymbol{g}, 0\right)$, and the uncertainty in $\boldsymbol{f}$ occurs because we suppose that the spring constant $k(t)$ is known only as an element in the set [150, 250] N/m and can drift over time. The drift of the spring constant is simulated by a random walk with reflecting boundaries at 150 and 250 given by $k(t)=k(t-1)+\eta(t)$ where $\eta(t)$ is distributed

\footnotetext{
${ }^{13}$ Since Bupp's controller does not explicitly consider the cost function

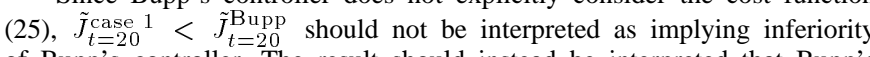
of Bupp's controller. The result should instead be interpreted that Bupp's controller exhibits similar performance as the satisficing controller.
}

uniformly over $\{-2,2\}$. For any $k \in[150,250]$ there exists a corresponding $f^{\gamma}, \gamma \in \Gamma=[150,250]$ such that all belief is placed on $k=\gamma$ where $\theta^{\gamma}=\left(\boldsymbol{f}^{\gamma}, \boldsymbol{g}, 0\right)$ and $f_{\theta}^{\gamma}(\theta)=\delta\left(\theta-\theta^{\gamma}\right)$. Simulation results for $b^{\text {case } 2}=3$ are shown in Fig. 3 and indicate that, despite the drifting spring constant, the controller regulates the cart about the origin with $\tilde{J}_{t=20}^{\text {case } 2}=273.9$. Notice the small angular offset of approximately 0.025 radians which decreases approximately 0.001 radians every second. This terminal offset contributes significantly to the resulting cost and can be decreased by increasing the $Q$-weighting for $\psi$, but only in exchange for less damping.

For case three, $\theta^{\gamma}=\left(\boldsymbol{f}, \boldsymbol{g}, v^{\gamma}\right)$. The disturbance $v$ is a nonwhite random sequence generated by an autoregressive moving-average (ARMA) model given by

$$
A(q) v(t)=B(q) e(t) .
$$

where we have adopted the notation of [49] in defining the transfer operators $A(q)$ and $B(q)$ and where $e(t)$ is an independently identically distributed zero mean Gaussian noise sequence with variance equal to two. In practice, $A(q)=$ $1+0.5 q^{-1}$ and $B(q)=1-0.3 q^{-1}$. Using system identification methods, we identify $H(q)=B(q) / A(q)$ from which $v(t)$ is predicted from past observations of the disturbance. We restrict attention to a set of model structures which uniquely predict the next value of $v(t)$. Associated with each model structure is the best model $H^{\gamma}(q)$, which yields the prediction ${ }^{14} \hat{v}(t \mid t-$ $1)^{\gamma}=H^{\gamma}(q) e(t)$ of the disturbance $v(t)$. Thus, $\theta^{\gamma}=$ $\left(\boldsymbol{f}, \boldsymbol{g}, H^{\gamma}(q) e\right)$ and the corresponding set of belief functions is the set $\mathcal{F}=\left\{f_{\theta}^{\gamma}: f_{\theta}^{\gamma}=\delta\left(\theta^{\gamma}-\theta\right)\right\}$.

Continuing case three, models of $A(q)$ and $B(q)$ were identified using MATLAB's System Identification Toolbox from four model structures by applying a pseudorandom input signal $u(t) \in\{-1,+1\}$ to the discretized equations (23)-(24) for a Gaussian white noise sequence $e(t)$ and estimating $v(t)$ by: 1) predicting $\hat{\boldsymbol{x}}(t+1)$ using known $u(t) ; 2)$ determining $\boldsymbol{g}(\boldsymbol{x}(t)) v(t)=\boldsymbol{x}(t+1)-\hat{\boldsymbol{x}}(t+1)$; and 3) finding $v(t)$ from

\footnotetext{
${ }^{14} \hat{v}(t \mid t-1)$ denotes an estimate of the disturbance at time $t$ given past observations $v(t-1), v(t-2), \cdots v(0)$.
} 
TABLE I

IDENTIFIED Disturbance Models

\begin{tabular}{c|c|c}
\hline Class & $A(q)-1$ & $B(q)-1$ \\
\hline \hline Actual & $0.5 q^{-1}$ & $-0.3 q^{-1}$ \\
\hline ARMA $_{1}$ & $0.5033 q^{-1}$ & $-0.2892 q^{-1}$ \\
\hline ARMA $_{2}$ & $1.0596 q^{-1}+0.2696 q^{-2}$ & $-0.2684 q^{-1}-0.1757 q^{-2}$ \\
\hline $\mathrm{AR}$ & $0.7792 q^{-1}+0.1776 q^{-2}$ & 0 \\
\hline $\mathrm{SS}$ & $0.5023 q^{-1}$ & $-0.2909 q^{-1}$ \\
\hline ARMA $_{1}$ & $0.4980 q^{-1}$ & $-0.3231 q^{-1}$ \\
\hline ARMA $_{2}$ & $-0.1566 q^{-1}-0.3535 q^{-2}$ & $-0.9756 q^{-1}+0.1768 q^{-2}$ \\
\hline $\mathrm{AR}$ & $0.7981 q^{-1}+0.1923 q^{-2}$ & 0 \\
\hline $\mathrm{SS}$ & $0.4952 q^{-1}$ & $-0.3252 q^{-1}$ \\
\hline
\end{tabular}

$\boldsymbol{g}(\boldsymbol{x}(t)) v(t)$. Four model structures were chosen: a first-order $\left(\mathrm{ARMA}_{1}\right)$, a second-order $\left(\mathrm{ARMA}_{2}\right)$, a second-order autoregressive (AR), and a second-order state space (SS) model identified using the prediction error method [49]. Two models were identified, one from each of two noise/input realizations, for each of these four model structures. This yields a total of eight identified models where $\Gamma=\{1,2, \cdots, 8\}$. Table I presents the model structures and the corresponding parameter estimates.

Simulation results using the robust strongly satisficing set are shown for $b^{\text {case } 3}=2$ in Fig. 3 and yield $\widetilde{J}_{t=20}^{\text {case } 3}=179.7$. Observe that the cart is effectively regulated about the origin despite disturbances. Also observe that to maintain similar performance, $b^{\text {case } 3}=2<3=b^{\text {case } 1}$. This decrease in $b$ is a result of the fact that as $\mathcal{F}$ grows to include differing models, $b$ must decrease for $S_{b}$ to be nonempty. For comparison purposes, it is insightful to omit the two frist order model classes since they have the same model structure as the actual disturbance. Doing so yields $\tilde{J}_{t=20}^{\text {case }} 3.1 \approx 179.7$ indicating that it is not necessary to have the actual disturbance model structure to produce acceptable performance.

\section{B. The Inverted Pendulum: Robustness and Defuzzification}

The inverted pendulum problem can be stated as follows. Control an inverted pendulum in a vertical plane with full circular freedom by applying a lateral force to the cart to which the pendulum is attached, while simultaneously regulating the position of the cart to any desired point. This problem has become a benchmark for nonlinear control design with examples from conventional control, fuzzy logic control, and other nonclassical control methods. For example, conventional controllers linearize the dynamics model of the pendulum in a small region within say $10^{\circ}$ of the vertical. FLC controllers include a FLC trained by a genetic algorithm, which has been shown to balance the pendulum $90 \%$ of the time if the pendulum is given a random initial position within $80^{\circ}$ of the vertical and a random initial velocity less than $80 \%$ [52]. An example of a particularly inventive nonclassical controller uses deterministic rules, fuzzy logic, and model-dependent information to control the pendulum with $360^{\circ}$ of motion on a constrained track [53].

A model-predictive satisficing controller for this problem has previously been presented [7], [8], which balances the pendulum and brings the cart to the origin for any initial cart position, cart velocity, pendulum angle, and pendulum angular

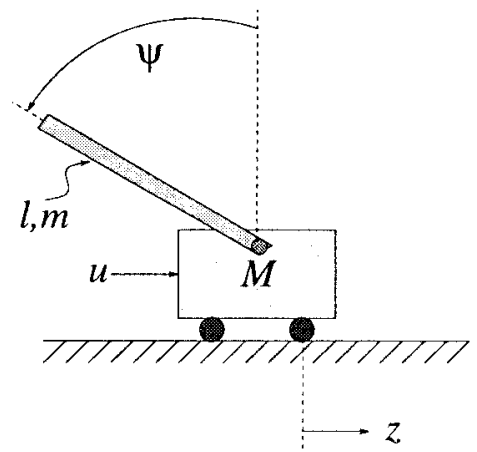

Fig. 4. Inverted pendulum on a cart. The pendulum has full rotational motion and must be regulated about the vertical while simultaneously keeping the cart near the origin.

velocity less than $180^{\circ}$ per sample time $T$. The approach employs predictions obtained from a nonlinear model of the plant dynamics, uses a single time step receding control horizon, and determines accuracy and liability using quadratic cost functions. A criticism of this design is the omission of either a stability argument or an appeal to expert rules. In this paper, we extend the previous results by: 1) employing expert information to generate the appropriate cost functions; 2) designing a controller with explicit attention to robustness; and 3) illustrating the flexibility of reduced-search defuzzifier design.

The inverted pendulum apparatus is illustrated in Fig. 4, where $M$ is the mass of the cart, $l$ is the length of the pendulum, $m$ is the mass of the pendulum, $\psi$ is the angle from vertical (measured counterclockwise), $z$ is the horizontal position of the cart, and $u$, the control input, is a lateral force applied to the cart. The continuous-time dynamical equations for this problem are

$$
\begin{aligned}
& \ddot{\psi}=\frac{(M+m) g \sin \psi-m l \cos \psi \sin \psi \dot{\psi}^{2}-\cos \psi u}{l\left(M+m \sin ^{2} \psi\right)} \\
& \ddot{z}=\frac{m l \sin \psi \dot{\psi}^{2}-m g \cos \psi \sin \psi+u}{M+m \sin ^{2} \psi}
\end{aligned}
$$

which can be easily written in the form of (17) with state variable $\boldsymbol{x}(t)=[\psi(t), z(t), \dot{\psi}(t), \dot{z}(t)]^{T}$ and which can be shown to satisfy the conditions in Section III. In the simulation results, we use Euler integration with a sample time of $T=$ $0.01 \mathrm{~s}$ and the following parameter values: $M=0.455 \mathrm{~kg}$, $l=0.61 \mathrm{~m}, U=[-1000,1000]$, and (nominally) $m=0.21$ $\mathrm{kg}$. For this problem, we consider the disturbance free case with uncertain pendulum mass where we use the simplified notation $\theta^{\gamma}=\boldsymbol{f}^{\gamma}$.

It is clear that, although the substates $\psi(t+1)$ and $z(t+1)$ are not explicit functions of $u(t)$, the substates $\dot{\psi}(t+1)$, $\dot{z}(t+1), \psi(t+2)$, and $z(t+2)$ are. Thus, we may identify the components of the state that are influenced by the current input $u(t)$ as the velocity vector $\boldsymbol{v}(t+1)=[\dot{\psi}(t+1), \dot{z}(t+1)]^{T}$ and position vector $\boldsymbol{p}(t+2)=[\psi(t+2), z(t+2)]^{T}$ yielding $\boldsymbol{\chi}(t)=[\boldsymbol{p}(t+2), \boldsymbol{v}(t+1)]^{T}$.

1) Specification of Attributes: We now turn attention to choosing $P, Q, R, \kappa_{A}$, and $\kappa_{L}$ in (19)-(22) to yield desirable results. We normalize the membership functions so that the 
maximum membership is unity and the minimum membership is zero (over the region of support $\mathcal{E}$ ). The corresponding normalizing constants are given by

$$
\begin{aligned}
\kappa_{A} & =\frac{1}{\max _{v \in \mathcal{E}} \Phi(v)-\min _{v \in \mathcal{E}} \Phi(v)} \\
\kappa_{L} & =\frac{1}{\max _{v \in \mathcal{E}} \Lambda(v)-\min _{v \in \mathcal{E}} \Lambda(v)} .
\end{aligned}
$$

The values in the cost matrices are determined by associating elements of these matrices with particular rules. For the first simulation result, the most discriminating defuzzifier defined in Theorem 2 is used. The flexibility of defuzzifier design is demonstrated in the second simulation result.

The inverted pendulum can be thought of as a regulator problem since the goal of the system is to regulate the system about a desired operating point. Since regulation is the purpose of the controller and since regulation cannot be precisely determined by considering only a single-step control horizon $(N=1)$, the set of accurate controls is fuzzy and, consequently, associated with a set-membership function. The accuracy of a control is dictated by the following rule: those controls for which $\chi$ is close to the origin (regulating point) have high accuracy. Since each element of $\boldsymbol{\chi}$ is equally important with respect to this purpose, we let $P=I$.

Liability is determined by an expert who not only specifies the structure of the cost matrix according the observed design principles but also tunes the numerical values in simulation. The quadratic cost function can be used because no more than second order relationships between elements of $\chi$ are required $^{15}$ (as evident from the following rule base). The following rules specify the design principles:

$A$ If cart position, pendulum angle, or velocities produced by a control are relatively large the control is liable.

$B$ If cart position and pendulum angle produced by the control have the same sign the control is liable. This reflects the expert understanding that when the pendulum is pointing to the left/right and the cart is also to the left/right the system is not very close to being balanced.

$C$ If cart velocity and pendulum velocity produced by the control have the same sign the control is liable. This reflects the expert understanding that when the pendulum is swinging the same direction that the cart is traveling, the system is either getting farther from being balanced or needs to avoid overshoot.

$D$ If a control requires a lot of energy then it is liable.

These rules dictate a block diagonal structure of the matrix $Q$. We identify the relationships between elements of $\boldsymbol{x}$ dictated by the rules by placing the appropriate rule $(1,2$, $3,4)$ in the matrices below. If no relationship exists, then the matrix element is zero.

$$
Q=\left[\begin{array}{cccc}
A & B & 0 & 0 \\
B & A & 0 & 0 \\
0 & 0 & A & C \\
0 & 0 & C & A
\end{array}\right] \quad R=D
$$

\footnotetext{
${ }^{15}$ For more complicated relationships, a more sophisticated inference engine is required.
}

Obviously, the relative weight of these rules will influence the system behavior. For example, if minimizing velocities is more important than minimizing positions, then the pendulum may never generate enough energy to move above the horizon. Such issues are resolved by tuning the weights to the nominal cart/pendulum system yielding [7]

$$
Q=\left[\begin{array}{cccc}
30 & 1 & 0 & 0 \\
1 & 0.3 & 0 & 0 \\
0 & 0 & 0.2 & 0.2 \\
0 & 0 & 0.2 & 0.2
\end{array}\right] \quad R=10^{-4}
$$

For the cart/pendulum system used in the simulation, the $Q$ values indicate that making the pendulum angle small (weight 30) is more important than keeping the cart near the origin (weight 0.3 ). They also indicate that keeping the cart position and pendulum angle opposite signs (weight 1 ) is more important than all other considerations except bringing the pendulum to vertical.

2) Results: In the simulations we consider two cases. The first case has no disturbances but does have an uncertain pendulum mass ( $\boldsymbol{f}$ from (17) is uncertain) where $\theta^{\gamma}=\boldsymbol{f}^{\gamma}$. This case forms an illustration of the application of robust satisficing receding horizon control. A second case is also presented when the plant has certain system parameters and no disturbances, where $\theta=\boldsymbol{f}$ with $\boldsymbol{f}$ known and fixed. This case illustrates two important aspects of our methodology: 1) that the defuzzifier design is flexible and 2) that reduced search alternatives are possible.

For case one $\theta^{\gamma}=f^{\gamma}$ and the uncertainty in the system model occurs because we suppose that the mass at time $t+1$ is given by $m(t+1)=m(t)+\eta(t)$ where $\eta(t)$ is distributed uniformly over $\{-0.01,0.01\}$. Suppose further that this random walk is restricted so that $m(t) \in[0.10,0.30]$ for all time. In the absence of other information, it is desirable to design a control such that for all ${ }^{16} m(t) \in[0.10,0.30]$ the plant performance is justifiable. For any $m \in[0.10,0.30]$ there exists a corresponding $\boldsymbol{f}^{\gamma}, \gamma \in \Gamma=[0.10,0.30]$ such that all belief is placed on $m=\gamma$ where $\theta^{\gamma}=f^{\gamma}$ and $f_{\theta}^{\gamma}(\theta)=\delta\left(\theta-\theta^{\gamma}\right)$.

Applying a robust satisficing fuzzy logic controller to this problem with $b=0.9$ yields the rotational and translational phase-plane performance illustrated in Fig. 5(a) and (b). The "o" symbol represents the initial conditions (the cart at the origin with the pendulum in the vertical down position) and the " $X$ " symbol represents the terminal conditions (the cart at the origin with the pendulum balanced in the vertical up position). The controller balances the pendulum while regulating cart position by swinging the pendulum back and forth while the cart oscillates around the origin. As the cart oscillates, the pendulum gathers momentum. In the translational and rotational phase planes, this motion is manifest as growing spirals. When the amplitude increases sufficiently, the oscillation ceases and the pendulum then converges to the vertical upright position. The cart then returns slowly to the origin. For

\footnotetext{
${ }^{16}$ The results presented in [7] for a randomized mass do not explicitly account for the unknown value of $m(t)$, but instead employ the known (albeit randomly time-varying) value of $m(t)$ via to produce acceptable performance via local model predictions.
} 


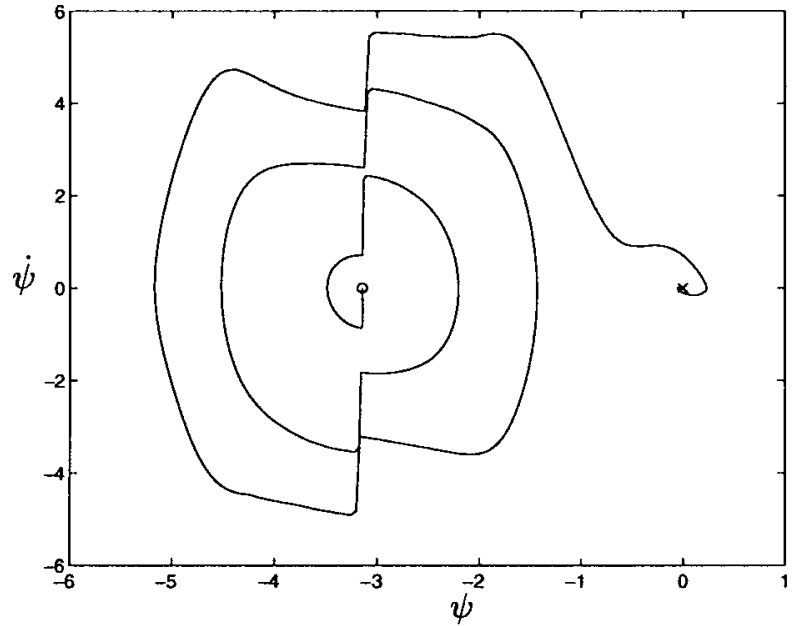

(a)

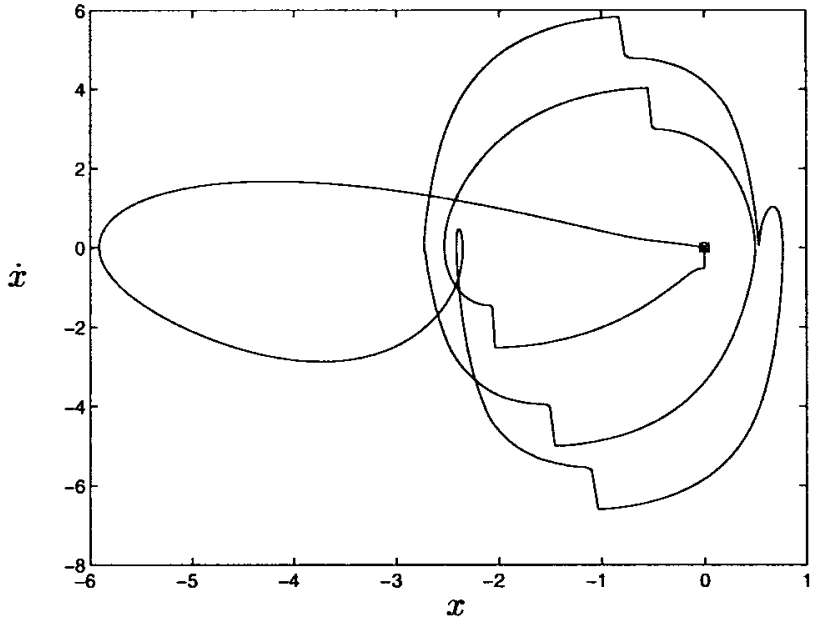

(b)

Fig. 5. Phase planes for the inverted pendulum for uncertain system. (a) Rotational pendulum angle (in radians per second and radians). (b) Translational cart position (in meters per second and meters).

these simulations, the most discriminating defuzzifier defined in Theorem 2 is used.

Observe that the perturbations $\eta(t)$ of the mass $m(t)$ are as large as $10 \%$. We can interpret this in two ways. First and most simply, this means that a large uncertainty is included in the discrete time system and that as designed, the controller is robust with respect to this uncertainty. Second, the large uncertainty in $m(t)$ can be interpreted as evidence that the discrete time control law would be appropriate (i.e., the controller handles significant errors between model-based predicted performance and simulated plant performance) for the continuous time system (the physical system was not available for testing).

For case two, $\theta=f$ is certain and a flexible reduced-search defuzzifier design is explored. ${ }^{17}$ When no closed-form solution can be found, applying maximizing defuzzifiers requires a search. Flexible reduced-search defuzzifying is important since, for example, applying a receding control horizon to many nonlinear control problems requires a planning horizon greater than one step (for example, to guarantee stability) and the resulting control law may not have a closed form. A search must therefore by applied and such search should not be computationally prohibitive. For the satisficing approach, a defuzzifying algorithm can be constructed that reduces search by "staying the course" with the current control when possible and otherwise selecting any satisficing solution. The algorithm for such search is as follows. Let $u(t)$ represent the current control. If $u(t) \in \mathcal{S}_{b}$, then no search needs to be done because $u(t+1)=u(t)$ is a satisficing solution. Otherwise, a search needs to be done because $u(t) \notin \mathcal{S}_{b}$. Using the randomized defuzzifier, this search randomly selects possible solutions until one is obtained that is satisficing. As soon as a satisficing solution is found, this solution is implemented. Because any element in the strongly satisficing set can be justifiably applied as a control, any defuzzifier that selects from this set can be

\footnotetext{
${ }^{17}$ For case one, the most discriminating defuzzifier was applied, but this is not the only choice. For example, applying a centroid defuzzifier produces a result nearly identical to the maximum defuzzifier.
}

used. To emphasize this point in case two, a simulation was performed using a defuzzifier which randomly (with uniform probability) selected a control from the strongly satisficing set for $m$ known and fixed. The rotational and translational phase planes for one such simulation with $b=0.9$ are presented in Fig. 6(a) and (b). Observe that the behavior produced by the random defuzzifier is qualitatively very similar to that produced by the most discriminating defuzzifier.

\section{CONCLUSIONS}

Conventional optimal control employs an explicit system model and assumes an implicit expert who defines a cost function and solves the resulting optimization problem. Conventional fuzzy control assumes an implicit model and employs an explicit expert to define solvable performance criteria. In this paper, we employ an explicit model to predict local plant behavior and an explicit expert to transform these local model predictions into global evaluations of gains and losses. This formulation implements these characteristics in a three step process: 1) explicit models are used to infer local consequences from observations; for problems where complexity and uncertainty dictate that long range computable and precise plant descriptions are infeasible, local predictions may be available and applicable to generating a useful solution; 2) explicit expert rules are used to infer valuations from local consequences; such inferences are applicable to problems where mathematically stable control laws are not trivially constructed, but where local information can be related to global performance; and 3) finally, satisficing decision theory infers control actions from valuations by combining independent assessments of goal achievement gains with proximate performance losses.

Since the relation between local plant predictions and global system performance is nonstatistically uncertain, fuzzy descriptions are required. Given these fuzzy descriptions, it seems unreasonable to suppose that a single unique cost function unambiguously specifies a best global performance. Instead the error-avoiding principle associated with compara- 


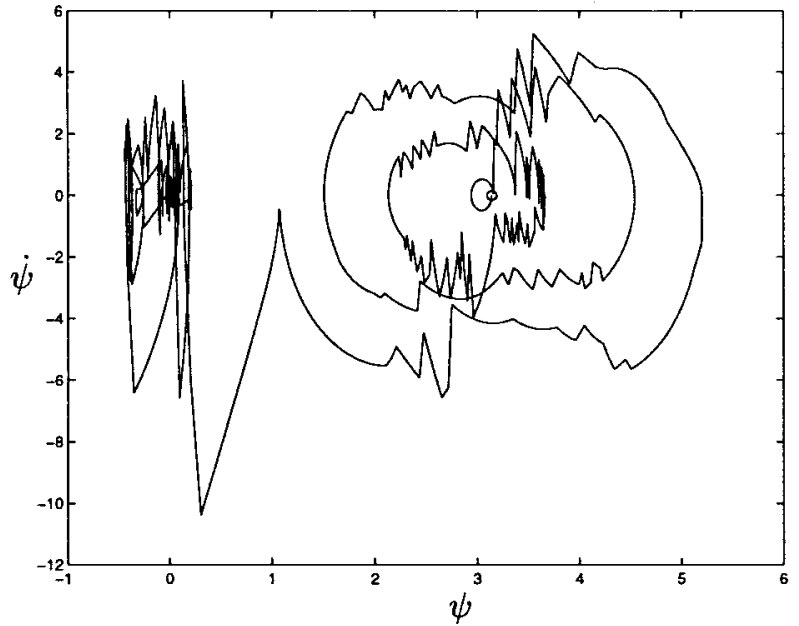

(a)

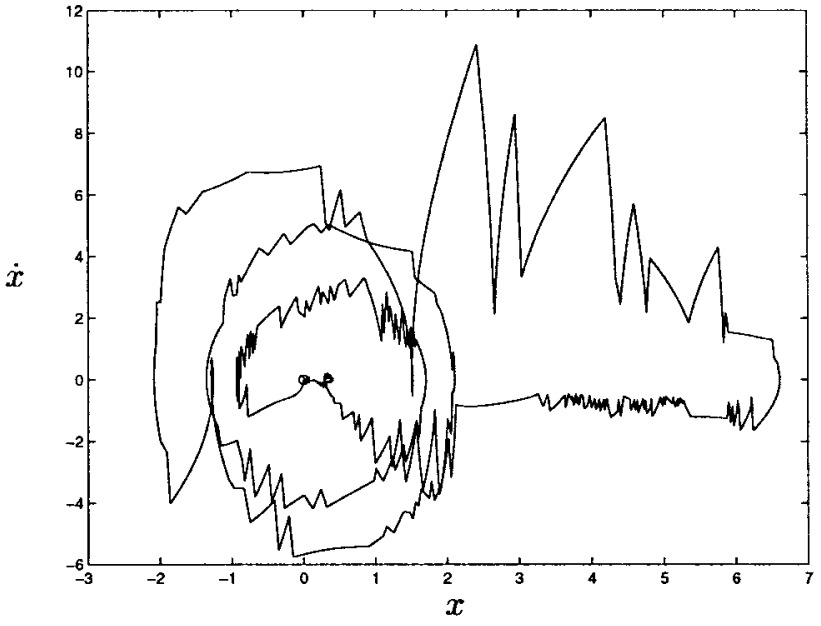

(b)

Fig. 6. Phase planes for the inverted pendulum with constant mass and reduced search/random defuzzifier. (a) Rotational pendulum angle (in radians per second and radians). (b) Translational cart position (in meters per second and meters).

tive rationality can be used to produce the set of satisficing system controls. This application of the satisficing principle determines when a control solution is justified given the observed evidence. When combined with the domination principle which eliminates control solutions when superior solutions exist, the resulting set-valued formulation facilitates both flexible defuzzifier design as well as robustness with respect to uncertain models and nonwhite disturbances.

In this paper, we have presented a synthesis procedure for designing fuzzy logic controllers. This procedure is derived from the SSDT-based model predictive approach, but which has been extended to: 1) appropriately employ the power of expert knowledge and 2) account for robust performance in the presence of uncertainty. We have demonstrated the synthesis of satisficing fuzzy logic controllers for two discrete time nonlinear problems. The synthesis procedure is based on identifying rules and cost functions, which simultaneously use objective (albeit possibly uncertain) local one-step predictions derived from mathematical models and subjective (expert) interpretation of the global system consequences. These sources of knowledge are used to identify the accuracy and liability of a possible control and, though the provable stability of such systems remains an open research question, expert knowledge was used to generate feasible controllers. Using the RTAC, we demonstrated robustness with respect to nonwhite disturbances. Using the inverted pendulum, we demonstrated that successful control of the model-based problem is flexible with respect to the defuzzification procedure. Additionally, using each of the problems, we demonstrated robustness properties with respect to model uncertainty.

\section{REFERENCES}

[1] A. Meystel, Autonomous Mobile Robots. Singapore: World Scientific, 1991.

[2] Y. C. Ho, "Heuristics, rules of thumb, and the 80/20 proposition," IEEE Trans. Automat. Contr., vol. 39, pp. 1025-1027, May 1994.

[3] Y.-C. Ho, "On the numerical solutions of stochastic optimization problem," IEEE Trans. Automat. Contr., vol. 42, pp. 727-729, May 1997.
[4] W. C. Stirling, M. A. Goodrich, and R. L. Frost, "Procedurally rational decision-making and control," IEEE Contr. Syst. Mag., vol. 16, pp. 66-75, Oct. 1996.

[5] L. A. Zadeh, "What is optimal?," IRE Trans. Inform. Theory, vol. 4, no. 1, p. 3, Mar. 1958.

[6] M. R. Endsley, "The role of awareness in naturalistic decision making," in Naturalistic Decision Making, C. E. Zsambok and G. Klein, Eds. Hillsdale, NJ: Erlbaum, 1997, ch. 26, pp. 269-283,

[7] M. A. Goodrich, W. C. Stirling, and R. L. Frost, "A theory of satisficing decisions and control," IEEE Trans. Syst., Man, Cybern.—Part A: Syst. Humans, vol. 28, pp. 763-779, Nov. 1998.

[8] _ "A satisficing approach to intelligent control of nonlinear systems," in Proc. IEEE Int. Symp. Intell. Contr., Dearborn, MI, Sept. 1996.

[9] H. A. Simon, "A behavioral model of rational choice," Quart. J. Econ., vol. 59 , pp. $99-118,1955$.

[10] _ The Sciences of the Artificial, 3rd ed. Cambridge, MA: MIT Press, 1996.

[11] I. Levi, The Enterprise of Knowledge. Cambridge, MA: MIT Press, 1980.

[12] J. M. Mendel, "Fuzzy logic systems for engineering: A tutorial," Proc. IEEE, vol. 83, pp. 345-347, Mar. 1995

[13] S. Roychowdhury and B.-H. Wang, "Cooperative neighbors in defuzzification," Fuzzy Sets Syst., vol. 78, no. 1, pp. 37-49, Feb. 1996.

[14] M. A. Goodrich, W. C. Stirling, and R. L. Frost, "Model predictive satisficing fuzzy logic control," Tech. Rep. CBR TR 98-3, Nissan Cambridge Basic Res., Nissan Res. Development Inc., Cambridge, MA, 1998

[15] P. B. Sistu and B. W. Bequette, "Nonlinear model-predictive control: Closed-loop stability analysis," AIChE J., vol. 42, no. 12, pp 3388-3042, Dec. 1996

[16] J. Richalet, "Industrial applications of model based predictive control," Automatica, vol. 29, pp. 1251-1274, 1993.

[17] D. Q. Mayne and H. Michalska, "Receding horizon control of nonlinear systems," IEEE Trans. Automat. Contr., vol. 35, pp. 814-824, 1990.

[18] P. O. M. Scokaert, J. B. Rawlings, and E. S. Meadows, "Discrete-time stability with perturbations: Application to model predictive control," Automatica, vol. 33, no. 3, pp. 463-470, Mar. 1997.

[19] P. O. M. Scokaert and D. Q. Mayne, "Min-max feedback model predictive control for constrained linear systems," IEEE Trans. Automat. Contr., vol. 43, pp. 1136-1142, Aug. 1998.

[20] P. O. M. Scokaert and J. B. Rawlings, "Constrained linear quadratic regulation," IEEE Trans. Automat. Contr., vol. 43, pp. 1163-1169, Aug. 1998.

[21] K.-W. Li, I. B. Turksen, E. J. Davison, and K. C. Smith, "Stabilization of unstable and unintuitive plants by fuzzy control," IEEE Trans. Syst., Man, Cybern._Part B, vol. 27, pp. 55-67, Feb. 1997.

[22] S. K. Tso and Y. H. Fung, "Methodological development of fuzzylogic controllers from multivariable control," IEEE Trans. Syst., Man, Cybern.-Part B, vol. 27, pp. 566-572, June 1997.

[23] K. Tanaka and T. Kosaki, "Design of a stable fuzzy controller for an articulated vehicle," IEEE Trans. Syst., Man, Cybern.-Part B, vol. 27 , 
pp. 552-558, June 1997.

[24] L. X. Wang, "Stable adaptive fuzzy controllers with application to inverted pendulum tracking," IEEE Trans. Syst., Man, Cybern.—Part $B$, vol. 26, pp. 677-691, Oct. 1996.

[25] R. F. Popoli and J. M. Mendel, "Heuristically constrained estimation for intelligent signal processing," in Advances in Geophysical Data Processing, M. Simaan and F. Aminzadeh, Eds. Stamford, CT: JAI Press, 1989, vol. 3, pp. 107-134.

[26] _ "Estimation using subjective knowledge with tracking applications," IEEE Trans. Aerosp. Electron. Syst., vol. 29, pp. 610-623, July 1993.

[27] M. D. Mesarovic and Y. Takahara, "On a qualitative theory of satisfactory control," Inform. Sci., vol. 4, no. 4, pp. 291-313, Oct. 1972.

[28] T. Matsuda and S. Takatsu, "Characterization of satisficing decision criterion," Inform. Sci., vol. 17, no. 2, pp. 131-151, 1979.

[29] _ "Algebaric properties of satisficing decision criterion," Inform. Sci., vol. 17, no. 3, pp. 221-237, 17.

[30] S. Takatsu, "Decomposition of satisficing decision problems," Inform. Sci., vol. 22, no. 2, pp. 139-148, 1980.

[31] H. A. Simon, "Invariants of human behavior," Апnи. Rev. Psych., vol. 41, pp. 1-19, 1990.

[32] G. Gigerenzer and D. G. Goldstein, "Reasoning the fast and frugal way: Models of bounded rationality," Psych. Rev., vol. 103, no. 4, pp. 650-669, 1996

[33] C. E. Zsambok and G. Klein, Eds., Naturalistic Decision Making. Hillsdale, NJ: Erlbaum, 1997.

[34] L. A. Zadeh, "Maximizing sets and fuzzy markoff algorithms," IEEE Trans. Syst., Man, Cybern.—Part C: Applicat. Rev., vol. 28, pp. 9-15, Feb. 1998.

[35] M. Slote, Beyond Optimizing. Cambridge, MA: Harvard Univ. Press, 1989.

[36] 1998 NIPS Workshop Simple Inference Heurist. vs. Complex Decision Mach., Breckenridge, CO, Dec. 1998.

[37] "Special issue on higher order uncertainty," IEEE Trans. Syst., Man, .Cybern.-Part A: Syst. Humans, May 1996

[38] H. E. Kyburg Jr. and M. Pittarelli, "Set-based Bayesianism," IEEE Trans. Syst., Man, Cybern.—Part A: Syst. Humans, vol. 26, pp. 324-339, May 1996.

[39] __ "Bayesian and non-Bayesian evidential updating," Artif. Intell., vol. 31, pp. 271-293, 1987.

[40] P. L. Combettes, "The foundations of set theoretic estimation," Proc. IEEE, vol. 18, pp. 181-208, Feb. 1993.

[41] J. M. Maciejowski, Multivariable Feedback Design. Reading, MA: Addison-Wesley, 1989.

[42] H. Huang, "Asymptotic tracking and disturbance rejection in uncertain nonlinear systems," IEEE Trans. Automat. Contr., vol. 40, pp. 1118-1122, June 1995

[43] A. C. Teng, "Robust optimal design for dynamic and feedback control systems-With an emphasis on structured uncertainties," Ph.D. dissertation, Brigham Young Univ., Provo, UT, Apr. 1992.

[44] M. A. Goodrich and E. R. Boer, "Semiotics and mental models: Modeling automobile driver behavior," in Proc. Joint Conf. Sci. Technol. Intell. Syst. ISIC/CIRA/ISAS'98, Gaithersburg, MD, Sept. 1998.

[45] P. C. Fishburn, Subjective expected utility: A review of normative theories," Theory Decision, vol. 13, pp. 139-199, 1981.

[46] D. Driankov, H. Hellendoorn, and M. Reinfrank, An Introduction to Fuzzy Control, 2nd ed. New York: Springer-Verlag, 1996.

[47] G. C. Mouzouris and J. M. Mendel, "Nonsingleton fuzzy logic systems: Theory and applications," IEEE Trans. Fuzzy Syst., vol. 5, pp. 56-71, Feb. 1997.

[48] T. Lotan, "Modeling discrete choice behavior based on explicit information integration and its application to the route choice problem," IEEE Trans. Syst., Man, Cybern.—Part A: Syst. Humans, vol. 28, pp. 100-114, Jan. 1998.

[49] L. Ljung, System Identification: Theory for the User. Englewood Cliffs, NJ: Prentice-Hall, 1987.

[50] R. T. Bupp, D. S. Bernstein, and V. C. Coppola, "A benchmark problem for nonlinear control design: problem statement, experimental testbed, and passive nonlinear compensation," presented at $A C C$, Seattle, WA, June 1995.

[51] Z.-P. Jiang, D. J. Hill, and J. Guo, "Stabilization and tracking via output feedback for the nonlinear benchmark system," Automatica, vol. 34, no. 7, pp. 907-915, 1998

[52] J. Kim, Y. Moon, and B. P. Zeigler, "Designing fuzzy net controllers using genetic algorithm," IEEE Trans. Contr. Syst., vol. 15, pp. 66-72, 1995

[53] M. deSylva and J. Vagners, "Hybrid fuzzy logic control to stabilize an inverted pendulum from arbitrary initial conditions," in 10th IEEE Int. Symp. Intell. Contr., Monterey, CA, 1995.

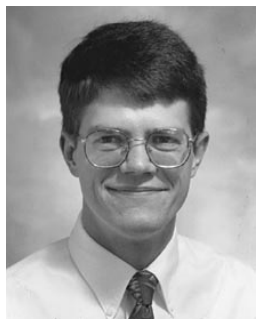

Michael A. Goodrich received the B.S. (cum laude), M.S., and Ph.D. degrees in electrical and computer engineering from Brigham Young University, Provo, UT, in 1992, 1995, and 1996, respectively.

From 1996 to 1998, he was a Research Associate with Nissan Cambridge Basic Research, Nissan Research and Development, Inc., Cambridge, MA, where he maintains status as a Visiting Scientist Since 1998 he has been with the Computer Science Department at Brigham Young University where he is an Assistant Professor. His research interests include modeling and controlling intelligent systems, decision theory, multiple-agent coordination, human-centered engineering, fuzzy logic, and estimation theory.

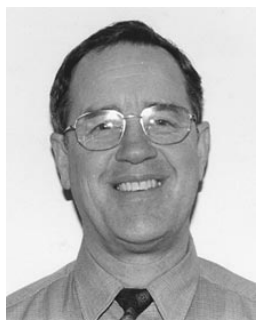

Wynn C. Stirling received the B.A. (honors magna cum laude, mathematics) and M.S. (electrical engineering) degrees from the University of Utah, Salt Lake City, Utah, and the Ph.D degree (electrical engineering) from Stanford University, Stanford CA, in 1969,1971 , and 1983, respectively.

From 1972 to 1975, he was with Rockwell International Corporation, Anaheim, CA, and from 1975 to 1984 he was employed by ESL, Inc., Sunnyvale, CA. Since 1984 he has been with Brigham Young University, Provo, UT, where he is a Professor in the Department of Electrical and Computer Engineering. His research interests include decision theory, control theory, estimation theory, and stochastic processes.

Dr. Stirling is a member of Phi Beta Kappa.

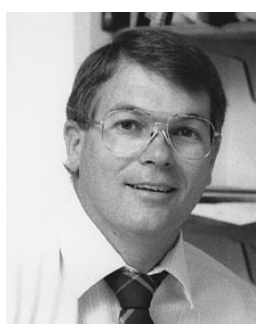

Richard L. Frost received the B.S. (physics, magna cum laude), M.S.E.E. (electrical engineering), and $\mathrm{Ph} . \mathrm{D}$. (electrical engineering) degrees, in 1975 , 1977, and 1979, respectively, all from the University of Utah, Salt Lake City.

$\mathrm{He}$ was with the Massachusetts Institute of Technology (MIT) Lincoln Laboratory, Cambridge, MA, from 1979 to 1981 , on the faculty of the University of Utah from 1981 to 1984 , and with the Communication Systems Division, Sperry (now L3 Communications), Salt Lake City, UT, from 1984 to 1987. In 1987 he joined the faculty at Brigham Young University. $\mathrm{He}$ is currently an Associate Professor in the Department of Electrical and Computer Engineering, Brigham Young University. His principal research interests include quantization and source coding and intelligent control. 